\title{
Clinical characteristics of health care workers infected with COVID-19 at the Single-Center Hospital in Turkey
}

\section{Corresponding author:}

Özlem Güdük, University of Sciences, Fatih Sultan Mehmet Training and Research Hospital, 34752 Istanbul, Turkey, e-mail: gudukozlem@gmail.com
Medical Research Journal 2021:

Volume 6, Number 1, 33-39 10.5603/MRJ.a2021.0005

Copyright (C) 2021 Via Medica

ISSN 2451-2591

e-ISSN 2451-4101

\begin{abstract}
Introduction: COVID-19 is a highly infectious disease characterized by different symptoms and varying severity from person to person. This study aims to identify the clinical characteristics of healthcare workers ( $\mathrm{HCWs)} \mathrm{who} \mathrm{have} \mathrm{been} \mathrm{infected} \mathrm{with} \mathrm{coronavirus} \mathrm{and-investigate} \mathrm{which} \mathrm{factors} \mathrm{affect} \mathrm{the} \mathrm{disease's} \mathrm{severity.}$ Materials and methods: In the presented study, 79 healthcare workers (HCWs) were recruited who had been infected with SARS-CoV-2, and working in a training and research hospital. Their data was examined in two groups as uncomplicated (without a computed tomography sign of pneumonia and respiratory rate $<24$ per minute, $\mathrm{SpO} 2>93 \%$ at room air) and pneumonia group in terms of the severity of the disease. The statistical analysis was performed by SPSS v.22.0 with a statistical significance of 0.05 .

Results: A total of $79 \mathrm{HCWs}$ with a mean age of $33.37 \pm 8.44$ years were enrolled in the study. They consisted of 47 female and 32 male participants. There were 50 patients in uncomplicated and 29 patients in the pneumonia group. A total of $14 \mathrm{HCWs}$ have been hospitalized with an average stay of $5.43 \pm 1,5$ days. The number of hospitalization between the groups was higher in the pneumonia group (n: 11) than in the uncomplicated group (n: 3$)(38 \%$ vs. $6 \% ; p<0.001)$. HCWs who work in areas in close contact with the patient (high-risk units) were more prone to be in the pneumonia group than those working in the other areas of the hospital [22/45 (48\%) vs. 7/27 (25\%); $p=0.019]$. There wasn't any significant difference between the groups in terms of age, gender, occupation, and the presence of chronic illness of workers ( $p$ $>0.05$ ). Sore throat and cough were the most common onset symptoms of the disease ( $34.2 \%$ and $31.6 \%$, respectively). There was no difference between the groups in terms of biochemical parameters. $(p>0.05)$. Conclusion: Healthcare workers are in the risk group for COVID-19 disease. HCWs working in high-risk units are more vulnerable.
\end{abstract}

Key words: COVID-19 outbreak, coronavirus, healthcare workers, disease's severity

Med Res J 2021; 6 (1): 33-39

\section{Introduction}

The novel Coronavirus disease (COVID-19) is a highly infectious disease leading to significant morbidity and mortality. The disease first appeared in Wuhan and spread rapidly throughout the world, causing havoc in the 21st century [1,2]. The first patient in Turkey was diagnosed on March 11, 2020. A total of 2.355.839 people got caught with the disease, and 23.325 people have died from that time to January 13 , 2020 , in the country [3]. In addition to affecting the entire society, the disease has also caused a significant number of deaths among healthcare workers (HCWs). According to the International Council of Nurses, it is estimated that 1,500 nurses in 44 countries died from COVID 19, and HCWs deaths from COVID 19 could amount to more than 20,000 worldwide [4].

Due to the high contagiousness of the disease, early diagnosis, isolation, and treatment are essential. Primary diagnostic uses include; computed tomography (CT) imaging and real-time reverse-transcriptase-polymerase chain reaction (RRT-PCR) [5]. In general, the radiographic features of the coronavirus are similar to those found in community-acquired pneumonia caused by other organisms. However, in terms of the CT image, COVID-19 pneumonia can be differentiated from non-COVID-19 pneumonia [6]. While COVID-19 pneumonia is more likely to have 
a peripheral distribution, ground-glass opacity, fine reticular opacity, and vascular thickening, it has less likely to have a central + peripheral distribution, pleural effusion, and lymphadenopathy [7].

Infection of the virus causes various effects on individuals. Although most people recover without requiring special treatment, the elderly and people with medical problems are more likely to develop illnesses seriously [8]. The most common clinical symptoms of the disease are fever, cough, shortness of breath, fatigue, myalgias, nausea/vomiting or diarrhoea, headache, weakness, and rhinorrhea [9], and radiological findings compatible with bilateral lung pneumonic infiltration. Besides, diarrhoea and vomiting are rare symptoms. Some patients may experience some degree of dyspnoea as an onset symptom. However, respiratory symptoms usually develop from several days to a week after the onset of the illness. Although the disease can be asymptomatic, pneumonia and severe acute respiratory tract infection may occur in $20-30 \%$ of the cases. Furthermore, renal failure and even death may develop in severe cases [10-12].

HCWs are inevitably at risk against COVID-19 disease. According to the Report of Amnesty International, at least 7,000 healthcare workers have died around the world after contacting COVID-19 until the beginning of September 2020 [13, 14]. Yet, little is known about the health status, basic demographics, severity of the disease, and computed tomography images of the healthcare workers receiving treatment for the disease. That's why more studies investigating the effects of COVID-19 disease on healthcare professionals are needed. This study aims to identify the clinical characteristics of HCWs who were infected with coronavirus and to investigate which factors affect the disease's severity.

\section{Materials and methods}

\section{Sample}

This is a single-centred, retrospective study of a group of HCWs infected with COVID-19 working at a training and research hospital. The demographic and clinical characteristics of data of the HCWs who had applied to the Occupational Health \& Safety Unit of the hospital with the diagnosis of coronavirus were collected from the hospital information system (HIS). The fact that the first employee applied on March 26, 2020 , was the starting date for collecting data. In the study, the authors included all the admissions of HCWs to the hospital for seven months from the onset of the disease. The data about the age, sex, occupation, chronic diseases (e.g., hypertension, diabetes, etc.) of the workers were collected, as were their departments, symptoms, and laboratory test results (e.g., albumin, bilirubin, C-reactive protein, D-dimer, etc.) at the onset, radiologic assessments of chest $\mathrm{CT}$, and hospitalization status.

We classified healthcare workers in terms of their clinical condition. For this purpose, the authors used "the COVID-19 (SARS-CoV-2 Infection) Adult Patient Treatment Guideline" set by Scientific Advisory Board working under the Turkish Health Ministry. According to the guideline, people are categorized into three groups. These are uncomplicated patients, patients with pneumonia, and patients in need of intensive care [15]. - Uncomplicated patients:

- Fever, muscle/joint pain, cough, and sore throat without respiratory distress (respiratory rate $<24$ per minute, SpO2 $>93 \%$ at room air),

- Patients with normal chest x-ray and/or lung tomography.

- Patients with pneumonia:

- Fever, muscle/pains, cough and sore throat, respiratory rate $<30$ per minute, SpO2 level $>90 \%$ in room air,

- Patients with signs of mild to moderate pneumonia on chest radiography or tomography.

- Patients in need of intensive care:

- Dyspnoea and respiratory distress,

- Respiratory rate $\geq 30$ per minute,

- $\mathrm{PaO} 2 / \mathrm{FiO} 2<300$,

- Oxygen need increase in follow-up

- $\mathrm{SpO} 2<90 \%$ or $\mathrm{PaO} 2<70 \mathrm{mmHg}$ despite $5 \mathrm{~L} / \mathrm{min}$ oxygen therapy,

- Hypotension (systolic blood pressure $<90 \mathrm{mmHg}$ and a decrease from usual SBP more than $40 \mathrm{mmHg}$ and mean arterial pressure $<65 \mathrm{mmHg}$, tachycardia > 100/min,

- Acute kidney damage, acute liver function test disorder,

- Patients with the development of acute organ dysfunction such as confusion, acute bleeding diathesis, and immunosuppression,

- High Troponin level and arrhythmia,

- Lactate $>2 \mathrm{mmol}$,

- Patients who meet the criteria for the presence of skin disorders such as capillary return disorder are evaluated to be treated in the intensive care unit.

In this study, the category of "patients in need of intensive care" was not udes since none of the workers needed intensive care treatment. Thus, healthcare workers were divided into two groups; (a) patients with typical CT signs and only positive PCR tests under the "uncomplicated" category (b) patients with CT results consistent with signs of pneumonia under the "pneumonia" category. 
Table 1. Demographic data of the patients

\begin{tabular}{|c|c|c|c|c|c|}
\hline \multicolumn{2}{|l|}{ Variable } & Total & Uncomplicated & Pneumonia & p-value \\
\hline \multicolumn{2}{|c|}{ Age: mean \pm SD (range) } & $34.37 \pm 8.4(20-57)$ & $32.26 \pm 7.22(20-52)$ & $36.28 \pm 9.39(22-57)$ & 0.189 \\
\hline \multicolumn{2}{|c|}{ Hospitalization: patients (\%) } & $14(18 \%)$ & $3(6 \%)$ & $11(38 \%)$ & 0.000 \\
\hline \multirow[t]{2}{*}{ Gender } & Female & $47(59.5)$ & $33(66)$ & $14(48.3)$ & 0.191 \\
\hline & Male & $32(40.5)$ & $17(34)$ & $15(51.7)$ & \\
\hline \multirow[t]{3}{*}{ Occupation } & Doctor & $18(22.8)$ & $12(24)$ & $6(20.7)$ & 0.944 \\
\hline & Nurse & $29(36.7)$ & $18(36)$ & $11(37.9)$ & \\
\hline & Others & $32(40.5)$ & $20(40)$ & $12(41.4)$ & \\
\hline \multirow[t]{2}{*}{ Units } & High risk & $45(57)$ & $23(46)$ & $22(75.9)$ & 0.019 \\
\hline & Low risk & $34(43.0)$ & $27(54)$ & $7(24.1)$ & \\
\hline
\end{tabular}

Units, where HCWs work, were evaluated under two groups as "high-risk units" and "low-risk units" in terms of the contact possibility with COVID-19 patients. Highrisk units consisted of the emergency department, the operating room, the anaesthesia clinic, and the clinics where COVID-19 patients were diagnosed and treated. Those other than these units were defined under the "low-risk units" category.

\section{Statistical analysis}

The data were evaluated with SPSS v.22.0 program. Mann Whitney $U$ test was used for two independent groups analysis, and Chi-Square tests (Yates', Fisher's Exact, Pearson Chi-Square) were used for categorical variables. Statistical significance was accepted at $p<0.05$ level.

The study was approved by the hospital Ethics Committee with the number 2020/28 and the date of $11 / 06 / 2020$.

\section{Results}

Between the periods of March 26, 2020, to November 02,2020 , a total of $79 \mathrm{HCWs}$ had been registered with COVID-19 diagnosis by the hospital's occupational health unit. These patients' sex distribution was as follows; 47 female (59.5\%), 32 male (40.5\%), were registered. The mean age of the total $\mathrm{HCWs}$ was $33 \pm 8.44$ years. Of the total participants, 50 (63.3\%) were in the uncomplicated group, and 29 (36.7\%) were in the pneumonia group.

A total of $14 \mathrm{HCWs}$ have been hospitalized with an average stay of $5.43 \pm 1,5$ days. Patients in the pneumonia group were more prone to be hospitalized as seen in
Table 1 (38\% vs. 6\%; $p<0.001$ ) All the inpatient cases investigated in this study discharged and none died.

There was no difference between the distribution of participants in terms of working as doctor, nurse or in other situations ( $p=0.944)$. In terms of the working units, most of the participants in the pneumonia group were working in high-risk units of the hospital. $22 / 29$ (75.9\%) vs. $7 / 29$ (24.1\%); $p=0.019$. Most of the employees in the pneumonia group consisted of workers working in the high-risk units. There weren't significant differences among the age and sex distribution of participants between uncomplicated and complicated groups.

In Table 2 were compared the chronic disease histories and symptoms during COVID-19 disease, depending on whether the participants were in the complicated or uncomplicated group.

When the workers' presences of chronic diseases were analysed, it was found that 16 healthcare workers diagnosed with nine different chronic diseases. Three of these workers had more than one disease. The most common chronic diseases among workers were hypertension, asthma, and diabetes mellitus. No significant relationship was found between the prognosis of COVID-19 and any of those nine chronic diseases $(p>0.05)$.

Sore throat, cough, malaise/weakness/fatigue, shortness of breath, and fever were the most common onset symptoms of HCWs. On the other hand, only one worker experienced stinging during breathing, and one worker had dysuria. None of the signs \& symptoms had a significantly important difference between the groups $(p>0.05)$.

There was no significant difference between the groups in terms of their biochemical blood parameters $(p>0.05)($ Tab. 3). 
Table 2. Chronic illness history of participants and symptoms of participants during the period of having COVID-19 infection

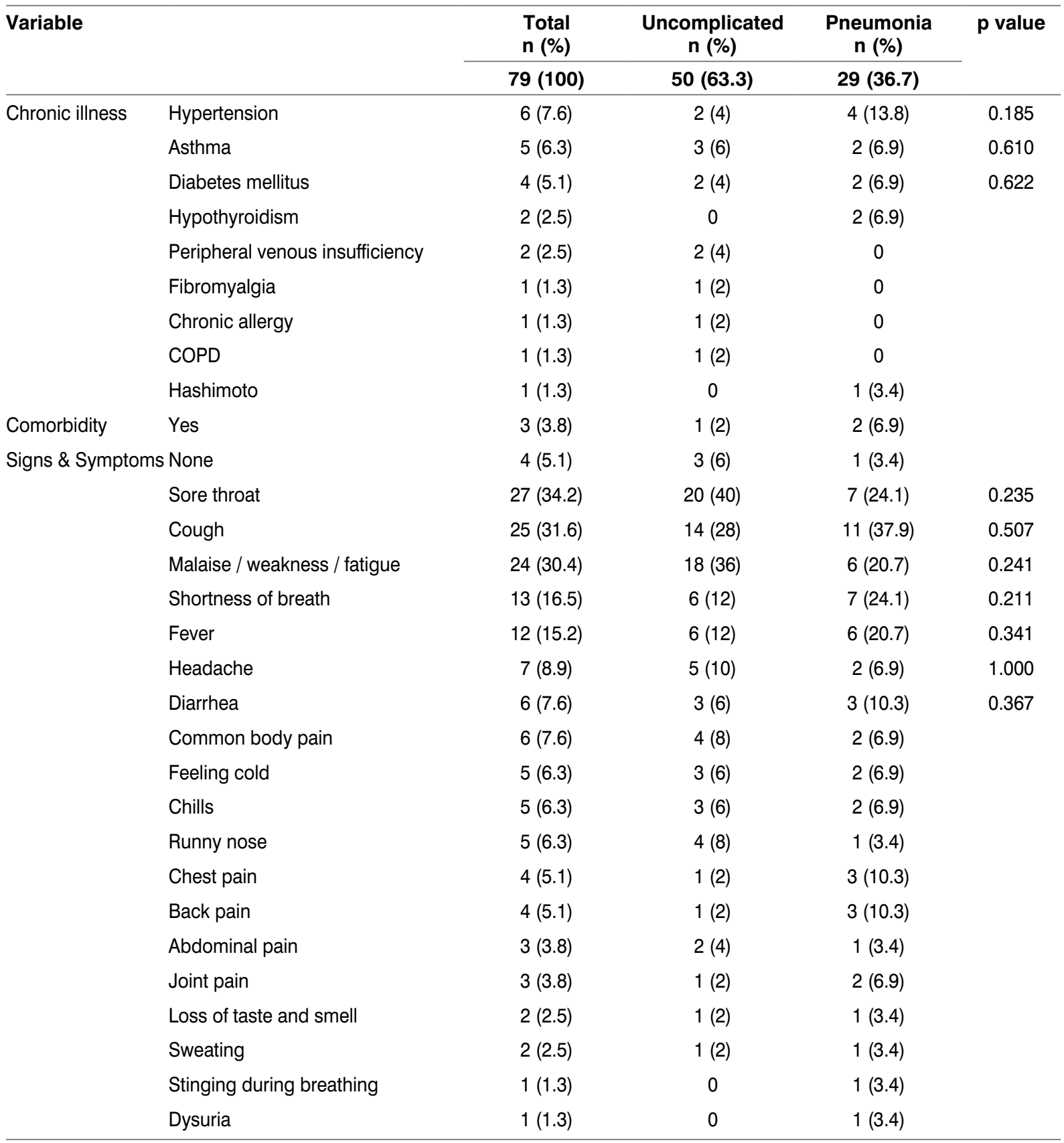

COPD - chronic obstructive pulmonary disease

\section{Discussion}

In this study, the clinical characteristics of healthcare workers diagnosed with coronavirus over seven months in a training and research hospital were evaluated according to the severity of the disease. Employees were examined in two groups (uncomplicated, pneumonia). Of the total participants, $63.3 \%$ were in the uncomplicated group, $36.7 \%$ in the pneumonia group. According to personal data (age, gender, job, unit of work, presence of chronic disease), the differences between the two groups and the initial symptoms and blood test results related to COVID-19 were examined.

It was found that the number of female workers was higher among diagnosed workers. Various studies find the different rates of female and male healthcare workers getting infected with COVID-19. In some studies, similar to ours, the rate of females was higher than males $[16,17]$. It is thought that the higher number of female employees in hospitals, in general, may affect 
Table 3. Comparison of the blood parameters of the groups

\begin{tabular}{|c|c|c|c|c|c|}
\hline & \multirow[t]{2}{*}{ Parameters } & \multirow{2}{*}{$\begin{array}{c}\text { Uncomplicated } \\
\text { n (\%) } \\
\text { Median }\end{array}$} & \multirow{2}{*}{$\begin{array}{c}\text { Pneumonia } \\
\text { n (\%) }\end{array}$} & \multirow[t]{2}{*}{$\mathbf{Z}$} & \multirow[t]{2}{*}{ P-value } \\
\hline & & & & & \\
\hline & Albumin $[\mathrm{g} / \mathrm{L}]$ & 44.5 & 45.2 & -0.753 & 0.474 \\
\hline & Bilirubin [direct][mg/dL] & 0.20 & 0.29 & -1.121 & 0.218 \\
\hline & C-reactive protein [mg/dL] & 0.40 & 0.71 & -1.426 & 0.156 \\
\hline & D-dimer [ng/mL] & 0.40 & 75.18 & -0.943 & 0.346 \\
\hline & White blood cells [103/uL] & 6.30 & 7.10 & -0.605 & 0.545 \\
\hline & Red blood cells [106/uL] & 4.95 & 5.04 & -0.063 & 0.950 \\
\hline \multirow[t]{7}{*}{ Blood tests } & Hemoglobin [g/dL] & 14.1 & 15.4 & -0.614 & 0.539 \\
\hline & Hematocrit [\%] & 41.8 & 44.7 & -0.307 & 0.759 \\
\hline & Platelet [103/uL] & 220 & 222 & -0.036 & 0.971 \\
\hline & Mean platelet volume [fL] & 9.70 & 9.35 & -1.688 & 0.091 \\
\hline & Neutrophil [103/uL] & 4.20 & 4.45 & -0.388 & 0.698 \\
\hline & Lymphocyte [103/uL] & 1.70 & 2.05 & -0.959 & 0.338 \\
\hline & Neutrophil / Lymphocyte [\%] & 0.45 & 0.50 & -0.731 & 0.465 \\
\hline
\end{tabular}

this result. On the other hand, according to Wiersinga et al., most cohort studies found that approximately $60 \%$ of patients were male [9]. However, in another study, an approximately $1: 1$ ratio of male $(50.7 \%)$ and female COVID-19 patients was found [15]. In this context, it cannot be said that gender has a direct risk factor for developing the disease. Besides this, the authors did not find any significant difference between the groups in terms of gender.

It was found that the total healthcare workers' median age was $33 \pm 8.44$ years $(\min =20, \max =57)$. Previous studies found that the total infected healthcare workers' median age was lower than hospitalized workers and the whole population. Mani et al. found that the median age of positive employees was 40 years [19]. In another study, the patients were between 23 and 63 years old, and the median age was 35 years [16]. However, the median age of hospitalized healthcare workers was 49 years [17]. On the other hand, Wiersinga et al. made a review of some studies related to COVID-19. They found that the median age of hospitalized patients among the general population was between 47 and 73 years. They also found that $74 \%$ to $86 \%$ of these patients were over the age of 50 [9]. Another study examining all hospitalizations within a certain period found the median age to be 57 [18]. In light of this information, it is seen that the average age of healthcare workers is lower compared to the general population.

In the study, only fourteen $\mathrm{HCW}$ s received inpatient treatment; the others were followed at their home. The number of hospitalization between the groups was higher in the pneumonia group ( $n: 11)$ than in the uncomplicated group (n: 3) (38\% vs. 6\%; $p<0.001)$. Hospitalization time varies in different studies. While Kambhampati et al. found that the median length of hospitalization among healthcare workers with COVID-19 was 4 days (IQR $=3-9$ days) [17], Liu et al. found 12.5 days [16]. According to Sahu et al., the incidence of severe disease in health care workers (9.9\%) was significantly lower than its incidence among all COVID-19 positive patients (29.4\%). They also found that the mortality rate in healthcare workers was meagre $(0.3 \%)$. Compared to all patients' mortality, this rate is lower than the general population (2.3\%). In the study, it is stated that this situation can be explained by the fact that healthcare workers are younger and have less comorbidity compared to the whole society. Besides, the early access of healthcare workers to the health system and better knowledge of the disease process has a positive effect [20]. However, in another study, a substantial proportion of HCWs with COVID-19 had indicators of severe disease. $27.5 \%$ of them were admitted to an intensive care unit, $15.8 \%$ required invasive mechanical ventilation, and $4.2 \%$ died during hospitalization [17]. In the presented study, it was found that healthcare workers were at young ages in general, and only $20.3 \%$ of employees had a chronic disease, which can support this study's results. In a study that looks at this situation from another perspective, the authors claimed that because the number of healthcare workers diagnosed with or died because of COVID-19 is not systematically reported, the real impact of the disease on healthcare workers in a global context is unknown [21]. It will also be useful to consider this discourse. 
In the presented study, the distribution of employees in the groups was examined in terms of the units they work in. The ratio of employees working in the high-risk units in the uncomplicated group was approximately $1: 1$, while $3 / 4$ of the pneumonia group employees were working in the high-risk units. As a result of the analysis, the authors found a significantly important difference in the employees' clinical conditions according to the unit they worked in $(p=0.019)$. Workers working in the frontline are thought to be more likely to become infected. In this context, Mani et al. found that $65.4 \%$ of positive test results were from frontline healthcare workers. ${ }^{19}$ In another study, more than two-thirds $(67.4 \%)$ of healthcare workers hospitalized with COVID-19 had worked with direct patient contact [17]. On the other hand, in another study among the affected health care workers, they found that many infected workers $(77.5 \%)$ worked at general wards, and only $22.5 \%$ worked in the emergency department and ICU (17.5\% and 5\%, respectively) [1]. Also, the authors found that there was no statistically significant difference in the proportion of COVID-19 positive PCR detection between healthcare workers from high-risk areas involved in close contact with COVID-19 patients in comparison with clerical, administrative, or laboratory personnel without direct contact with patients [22].

No significant difference between the groups in terms of occupation ( $p>0.05$ ) was found. However, nurses were the most affected professionals (36.7\%). Similarly, some other studies found that nurses were more affected workers among all workers. Kambhampati et al. found the proportion of nursing-related professions among hospitalized healthcare workers to be $36.3 \%$ [17]. In another study, $67 \%$ of those infected healthcare workers consisted of nurses [16]. These results may be explained by the fact that nurses have the highest number of occupational groups in hospitals.

The comorbidity rate was $20.3 \%$ in the presented study, and the most common chronic diseases were hypertension, asthma, and diabetes mellitus. The authors did not find a significant relationship between the prognosis of COVID-19 and any of the chronic diseases $(p>0.05)$. Similar to the presented study, Liu et al. found that the number of employees with one or more comorbidities was low (13\%) [16]. These were listed as hypertension, uterine fibroids, diabetes, depressive disorder, thyroid nodules, or abdominal lymphatic tuberculosis according to their prevalence. In the study of Zhang et al., hypertension (30.0\%) and diabetes mellitus $(12.1 \%)$ were the most common comorbidities [18]. Additionally, they found that the chronic obstructive pulmonary disease rate was low (1.4\%). On the other hand, according to Kambhampati et al., $89.8 \%$ of the workers had at least one underlying medical condition. They found that obesity was the most commonly reported disease $(72.5 \%)$, hypertension (40.6\%), and diabetes $(30.9 \%)$ were the other common diseases [17]. The low number of comorbidity in the presented study can be explained by the employees' low age. On the other hand, the fact that hypertension and diabetes were the most common chronic diseases can be explained by that they have a high prevalence in our population [23].

It was found that the most common onset symptoms were sore throat, cough, malaise/weakness/fatigue, shortness of breath, and fever. On the other hand, stinging in breathing and dysuria were very rare. Symptoms of the disease did not differ according to the severity of the disease. None of the signs \& symptoms had a significantly important difference between the groups ( $p>0.05$ ). In many studies, the most common clinical symptoms were fever, cough, fatigue [9, 10, 16-19, 24]. Also, it was found that shortness of breath [17], and headache (59.5\%), muscle aches (54.1\%), sore throat (50.8\%) were other common symptoms [19]. On the other hand, myalgia, dyspnoea, headache, dizziness, abdominal pain, diarrhoea, nausea, loss of appetite, difficulty breathing or chest tightness, chill, chest pain, and vomiting were less common symptoms [9, 10, 16, 18, 24]. Although the cause is not known precisely, it is known that the intestinal flora may change in COVID-19 patients [25]. Zhang et al. found that gastrointestinal symptoms were $39.6 \%$ among patients [18]. Another study had 15\%-39\% nausea/vomiting or diarrhoea symptoms [9]. However, according to Cetintepe and Ilhan, diarrhoea (3.7\%) and vomiting (5.0\%) are less common symptoms [10]. In a different study, $10.1 \%$ of the patients experienced diarrhoea and nausea 1 to 2 days before developing fever and dyspnoea [1]. Therefore, healthcare professionals need to consider these rare symptoms as well.

According to Wiersinga et al., the common laboratory abnormalities in hospitalized patients include lymphopenia, elevated inflammatory markers (e.g., erythrocyte sedimentation rate, C-reactive protein, ferritin, tumour necrosis factor-a, IL-1, IL-6), and abnormal coagulation parameters (e.g., prolonged prothrombin time, thrombocytopenia, elevated D-dimer, low fibrinogen) [9]. In another study, the blood counts of $17 \%$ of cases showed leukocytopenia, and only $2 \%$ showed leukocytosis on admission; $34 \%$ of the patients presented with lymphocytopenia and $11 \%$ presented thrombocytopenia. Elevated C-reactive protein and amyloid $A$ levels were presented in $45 \%$ and $59 \%$ of cases, respectively. Elevated levels of alanine aminotransferase and aspartate aminotransferase were less common. Only $3 \%$ of cases had abnormal procalcitonin serum levels. Notably, 47 (80\%) of cases had high levels of IL-6. However, most patients demonstrated normal levels of D-dimer, creatinine, and creatine kinase [16]. Zhang et al. investigated the patients into two groups (non-severe and severe). In their study, lymphopenia and eosinopenia were observed in most patients. Blood eosinophil counts correlate positively with lymphocyte counts in severe and non-severe patients after hospital 
admission. Significantly higher D-dimer levels, C-reactive protein, and procalcitonin were associated with severe patients compared to non-severe patients. More comorbidities, higher median values of leukocyte count, D-dimer, CRP, PCT, and lower lymphocyte percentage were found in severe cases, compared to non-severe cases. No difference was identified for the occurrence rates of most signs and symptoms between non-severe and severe patients [18]. In the presented study, the authors investigated albumin, direct bilirubin, C-reactive protein, D-dimer, white blood cells, red blood cells, haemoglobin, hematocrit, platelet, mean platelet volume, neutrophil, lymphocyte levels. Opposite of the previous studies, they did not find any significant high levels of these blood parameters. There was no significant relationship between the groups regarding their blood test results $(p>0.05)$. In the authors' opinion, this result may be explained by none of the workers stayed in the intensive care unit, and also, there was not any death result in their study.

\section{Conclusion}

COVID-19 is a highly contagious pandemic that the whole world has been struggling with for the last year. Healthcare workers are in the risk group for COVID-19 disease. HCWs working in high-risk units are more vulnerable. Identifying early symptoms and defining more vulnerable groups will help protect workers from disease.

\section{Limitations of the study}

This study has some limitations. First, this study was conducted with only 79 healthcare professionals who confirmed COVID-19 from a single hospital in Istanbul. Therefore, it can not be generalized for all healthcare professionals. Second, since it is a retrospective study, there was limited access to some data. More detailed data required for the study (e.g. employees' weight, smoking status, etc.) were not available at the analysis time. Thus, the study provides only a preliminary overview of a group of healthcare workers' epidemiological characteristics and clinical outcomes. More research is needed on this subject.

Conflict of interest: The authors declare that there is no conflict of interests regarding the publication of this paper.

\section{References}

1. Wang D, Hu Bo, Hu C, et al. Clinical Characteristics of 138 Hospitalized Patients With 2019 Novel Coronavirus-Infected Pneumonia in Wuhan, China. JAMA. 2020; 323(11): 1061-1069, doi: 10.1001/jama.2020.1585, indexed in Pubmed: 32031570.
2. Salazar de Pablo G, Vaquerizo-Serrano J, Catalan A, et al. Impact of coronavirus syndromes on physical and mental health of health care workers: Systematic review and meta-analysis. J Affect Disord. 2020; 275: 48-57, doi: 10.1016/j.jad.2020.06.022, indexed in Pubmed: 32658823.

3. https://covid19.saglik.gov.tr/TR-66935/genel-koronavirus-tablosu. html (23.10.2020)

4. ICN-The International Council of Nurses. October 28 2020. https:// www.icn.ch/news/icn-confirms-1500-nurses-have-died-covid-19-44countries-and-estimates-healthcare-worker-covid (24.12.2020).

5. Long $\mathrm{C}, \mathrm{Xu} \mathrm{H}$, Shen $\mathrm{Q}$, et al. Diagnosis of the Coronavirus disease (COVID-19): rRT-PCR or CT? Eur J Radiol. 2020; 126: 108961, doi: 10.1016/j.ejrad.2020.108961, indexed in Pubmed: 32229322

6. Ai T, Yang Z, Hou H, et al. Correlation of Chest CT and RT-PCR Testing for Coronavirus Disease 2019 (COVID-19) in China: A Report of 1014 Cases. Radiology. 2020; 296(2): E32-E40, doi: 10.1148/radiol.2020200642, indexed in Pubmed: 32101510.

7. Bai HX, Hsieh B, Xiong Z, et al. Performance of Radiologists in Differentiating COVID-19 from Non-COVID-19 Viral Pneumonia at Chest CT. Radiology. 2020; 296(2): E46-E54, doi: 10.1148/radiol.2020200823, indexed in Pubmed: 32155105.

8. https://www.who.int/health-topics/coronavirus \#tab=tab 1 (23.10.2020).

9. Wiersinga WJ, Rhodes A, Cheng AC, et al. Pathophysiology, Transmission, Diagnosis, and Treatment of Coronavirus Disease 2019 (COVID-19): A Review. JAMA. 2020; 324(8): 782-793, doi: 10.1001/jama.2020.12839, indexed in Pubmed: 32648899.

10. Cetintepe S, Ilhan M. COViD-19 Salgınında Sağlık Çalışanlarında Risk Azaltılması. Journal of Biotechnology and Strategic Health Research. 2020, doi: 10.34084/bshr.712539.

11. COVID-19 (SARS-CoV-2 Enfeksiyonu) Genel Bilgiler, Epidemioloji ve Tanı. https://covid19.saglik.gov.tr/Eklenti/39060/0/covid-19rehberigenelbilgilerepidemiyolojivetanipdf.pdf (23.10.2020).

12. Loeffelholz MJ, Tang YW. Laboratory diagnosis of emerging human coronavirus infections - the state of the art. Emerg Microbes Infect. 2020; 9(1): 747-756, doi: 10.1080/22221751.2020.1745095, indexed in Pubmed: 32196430.

13. Chu J, Yang N, Wei Y, et al. Clinical characteristics of 54 medical staff with COVID-19: A retrospective study in a single center in Wuhan, China. J Med Virol. 2020; 92(7): 807-813, doi: 10.1002/jmv.25793, indexed in Pubmed: 32222986.

14. Amnesty International. Global: Amnesty analysis reveals over 7,000 health workers have died from COVID-19. https://www.amnesty. org/en/latest/news/2020/09/amnesty-analysis-7000-health-workershave-died-from-covid19/ (23.10.2020)

15. T.C. Sağlık Bakanlığı. COVID-19 (SARS-CoV-2 Enfeksiyonu) Erişkin Hasta Tedavisi Bilimsel Danışma Kurulu Çalışması. https://covid19. saglik.gov.tr/TR-66926/eriskin-hasta-tedavisi.html (22.10.2020).

16. Liu J, Ouyang L, Guo Pi, et al. Epidemiological, Clinical Characteristics and Outcome of Medical Staff Infected with COVID-19 in Wuhan, China: A Retrospective Case Series Analysis., doi: 10.1101/2020.03.09.20033118.

17. Kambhampati A, O'Halloran A, Whitaker M, et al. COVID-19-Associated Hospitalizations Among Health Care Personnel — COVID-NET, 13 States, March 1-May 31, 2020. MMWR. Morbidity and Mortality Weekly Report. 2020; 69(43): 1576-1583, doi: 10.15585/mmwr.mm6943e3.

18. Zhang JJ, Dong X, Cao YY, et al. Clinical characteristics of 140 patients infected with SARS-CoV-2 in Wuhan, China. Allergy. 2020; 75(7): 1730-1741, doi: 10.1111/all.14238, indexed in Pubmed: 32077115.

19. Mani NS, Budak JZ, Lan KF, et al. Prevalence of COVID-19 Infection and Outcomes Among Symptomatic Healthcare Workers in Seattle, Washington. Clin Infect Dis. 2020 [Epub ahead of print], doi: 10.1093/cid/ciaa761, indexed in Pubmed: 32548613

20. Sahu AK, Amrithanand VT, Mathew R, et al. COVID-19 in health care workers - A systematic review and meta-analysis. Am J Emerg Med. 2020; 38(9): 1727-1731, doi: 10.1016/j.ajem.2020.05.113, indexed in Pubmed: 32738467.

21. PALA SC, METINTAS S. COVID-19 Pandemisinde Sağlık Çalışanları. Eskişehir Türk Dünyası Uygulama ve Araştırma Merkezi Halk Sağlığı Dergisi. 2020, doi: 10.35232/estudamhsd.789806.

22. Folgueira M, Muñoz-Ruipérez C, Alonso-López M, et al. SARS-CoV-2 infection in Health Care Workers in a large public hospital in Madrid, Spain, during March 2020. , doi: 10.1101/2020.04.07.20055723.

23. Satman İ, Alagöl F, Ömer B, et al. Türkiye Diyabet, Hipertansiyon, Obezite ve Endokrinolojik hastalıklar prevalans çalışması-II 2010.

24. Wang X, Liu W, Zhao J, et al. Clinical characteristics of 80 hospitalized frontline medical workers infected with COVID-19 in Wuhan, China. J Hosp Infect. 2020 [Epub ahead of print], doi: 10.1016/j. jhin.2020.04.019, indexed in Pubmed: 32302722.

25. Perisetti A, Gajendran M, Goyal H. Putative Mechanisms of Diarrhea in COVID-19. Clin Gastroenterol Hepatol. 2020; 18(13): 3054-3055, doi: 10.1016/j.cgh.2020.06.008, indexed in Pubmed: 32535231. 